\title{
Personalised information services for bikers
}

\section{Dávid Földes* and Csaba Csiszár}

Department of Transport Technology and Economics (KUKG), Faculty of Transportation Engineering and Vehicle Engineering (KJK), Budapest University of Technology and Economics (BME),

Müegyetem rkp. 1-3, H-1111 Budapest, Hungary

Email: foldes.david@mail.bme.hu

Email: csiszar.csaba@mail.bme.hu

*Corresponding author

\begin{abstract}
Modal share of cycling is growing; however, information services are to be significantly improved. Our research aim was to reveal, how the available real-time data can be used to support bikers and to ease decision making by provision of personalised information. We have identified the categories of biker information services and then the route planner applications have been analysed in order to point out the exemplary solutions. The attributes of mobility and information management, as well as their correspondences have been revealed in order to develop appropriate information services. We have carried out a questionnaire survey to identify bikers' habits and expectations towards information services. An analysis method has been elaborated which is applicable to determine the correspondences between mobility and information management attributes. The results are to be applied as bases during development of adaptive, personalised information application aiding decisions.
\end{abstract}

Keywords: behaviour; cycling; decision support; route planner; information management; information service; personalisation.

Reference to this paper should be made as follows: Földes, D. and Csiszár, C. (2018) 'Personalised information services for bikers', Int. J. Applied Management Science, Vol. 10, No. 1, pp.3-25.

Biographical notes: Dávid Földes received his MSc degree in Transportation Engineering from the Budapest University of Technology and Economics (BME), Hungary in 2016. He is studying as a $\mathrm{PhD}$ student at the same university. His research interests include intelligent and advanced transport systems with special regards to the future urban mobility issues, traveller behaviour (e.g., exanimation of personalisation opportunities) and decisions aided by information services. He is the author of eight scientific papers. He was awarded with Best Student Paper at ICALT 2016 conference in Krakow, Poland and is Second Place in National Students' Scientific Research Forum in 2015 with the theme 'Stop choosing algorithm development for personalised transport information applications'.

Csaba Csiszár received his MSc degree in Transportation Engineering, in 1998 and $\mathrm{PhD}$ in Transportation Science from the Budapest University of Technology and Economics (BME), Hungary, in 2002. He is an Associate Professor at Department of Transport Technology and Economics (KUKG), Faculty of Transportation Engineering and Vehicle Engineering (KJK) at the same university. His research interests include transport informatics and passenger transportation systems, with special regards to the system 
integration. He is the author of seven course books and 98 scientific papers. He has been a permanent invited member of Hungarian Academy of Sciences (MTA) Committee of Transport Sciences since 2009.

This paper is a revised and expanded version of a paper entitled 'Revealing correspondences between bikers' mobility and information management attributes' presented at IEEE International Conference on Advanced Logistics and Transport (IEEE ICALT'2016), Cracow, Poland, 1-3 June 2016.

\section{Introduction}

Each passenger transportation mode has specific mobility and information management properties and their correspondences are various. In this paper, we have focused on cycling. Modal share of cycling is growing in many urban regions because of biking infrastructure investments. The types of infrastructure that are advantageous for bikers:

- infrastructure where cycling is permitted

- cycle friendly infrastructure.

The latter one includes road types built especially for bikers (e.g., bike path, separated bike lane), or other road types where cycling is safe (e.g., low traffic road, cycle track, one-way streets with contraflow bicycle traffic).

However, development of biker information services lags behind extension of cycle friendly infrastructure. The availability and quality of information services (including advanced mobile applications) are to be improved with application of advanced technology. The information provision should be based on accurate data about current network conditions and environment (weather). Wide range of personalised settings and support of decision making is also required [initial hypothesis]. In case of several transportation modes, the travellers are supported by advanced infocommunication services (e.g., self-learning, adaptive, real-time applications).

The bicycle is a vehicle; therefore, it can be ridden even on the road. Riding among cars is dangerous; bikers are the most vulnerable travellers. Riding a bicycle on the pavement among pedestrians is forbidden in general; however, it is very frequent because of fear of riding on the road. Speed gaps may cause many conflicts both on road and pavement. We have collected the most important general attributes of cycling (Table 1).

The main objective of our research was to identify the development potential of biker information services in order to ease the decision-making and to improve the perceived quality of cycling. We have revealed the attributes of mobility and information management of biking, as well as their correspondences in order to develop and provide personalised information services. Individual setting activities of an application can be significantly reduced if biking preferences are derived from other available attributes. Mobility attributes: how the bikers behave, what kind of movements are typical during biking, what their preferences are. Information management attributes: what information services are required/preferred, how the information influences the bikers (e.g., decision-making); how the bikers transmit information about intended movements. 
Table 1 General attributes of cycling - advantages/disadvantages

\begin{tabular}{lll}
\hline Advantages & & \multicolumn{1}{c}{ Disadvantages } \\
\hline$\checkmark$ Competitive for short trip & $\times$ Conflict with pedestrians and road vehicles because of \\
& $\times$ speed gap \\
$\checkmark$ Part of multimodal journey & $\times$ Difficult to carry the bicycle on other transportation \\
& $\times$ means \\
$\checkmark$ Traffic congestion avoidable & $\times$ Storage on public place is less safe \\
& $\times$ Sensitive to air pollution \\
$\checkmark$ Storage is more simple & $\times$ Sensitive to weather \\
$\checkmark$ Promote sporty and healthy & $\times$ Highly sensitive to quality of road surface \\
lifestyle & $\times$ Sensitive to terrain \\
$\checkmark$ Affordable purchase and & $\times$ Carrying package is rather uncomfortable \\
operational cost & $\times$ Usage of individual information devices during riding is \\
& & difficult and dangerous \\
\hline
\end{tabular}

The remainder of the paper is structured as follows. In Section 2, relevant related works are reviewed. In order to reveal the correspondences between bikers' mobility and information management processes the model of biker's information management have been devised in Section 3. The exemplary functions and personalisation setting options have been identified by review and analysis of information services in Section 4. Data about bikers' personal and cycling attributes, information management (e.g., use of information devices/services, etc.), features of network and environment have been collected by questionnaire survey. Section 5 describes the data collection method. In Section 6, analysis method has been elaborated and applied in order to identify the correspondences between the attributes and to determine the strength of relationships. Section 7 summarises the structure of results highlighting some meaningful values. The relevant results have been discussed in Section 8. Finally, in Section 9, the conclusions are drawn and the future research directions are summarised.

\section{Related works}

Two types of 'sources' have been employed during research in order to identify bikers' habits and influencing factors. The experiences gained from our related previous research activities and from the up-to-date scientific results published in high quality journals. Our previous activity includes functions of applications aiding travel modes: public transport and walking (Földes and Csiszár, 2015), parking (Cserháti and Csiszár, 2016) and air transport (Karádi et al., 2015).

Several scientific papers and studies have been published pertaining to bikers' behaviour, attributes of bikers/cycling and route choice. The needed action plans to increase the number of cycling have been identified by Habib et al. (2014). They revealed with empirical investigations that latent perceptions of 'bikeability' and 'safety 
consciousness' directly influence the choice of biking. The bikers' individual choiceattributes, preferences have been analysed and an evaluation method for cycling routes has been developed by Ana et al. (2014). The results confirmed that safety aspects of the roads influence bikers' route choice. Braun et al. (2016) investigated how the decision to commute by bicycle was associated with cycling infrastructure, bike share availability, incentive programs and other attributes (e.g., public transport availability). They found that cycling and public transport are competing modes and presence of bicycle lane has a relatively small association with bicycle commuting. However, in another European study it has been found that cities with more extensive networks of bicycle lanes or paths can improve the modal share of cycling (Santos et al., 2013).

Several studies analysed the attractive and unattractive features of cycling with questionnaire surveys. The most unattractive features of a bicycle route are long travel time, high volume of motorised traffic, absence of continuous bicycle facility, high parking occupancy and long parking zones along the route (Bhat et al., 2015). The top five motivation factors are: routes away from traffic noise and pollution, routes with beautiful scenery, flat routes, bicycle path separated from traffic, shorter travel time by bike than by other modes (Winters et al., 2010). Choice of bicycle as a transportation mode is influenced by personal attitudes, emotions and social norms too (Passafaro et al., 2014). Investigating coherence between regularity and emotional sensitivity it has been found, that daily bikers are more sensitive to travel time, whereas inexpert, holiday bikers are more sensitive to environmental conditions (Stinson and Bhat, 2005). Tilahun et al. (2007) analysed preferences for different cycling facilities by stated preference survey. They found that respondents are willing to travel more to switch from an unmarked on-road facility with side parking to an off-road cycle track. In the case study (Ehrgott et al., 2012) different route choice methods regarding bikers and drivers of private vehicles have been analysed. It has been found that beside travel time other aspects are also important for bikers. A suitability index has been introduced which contains altogether 20 route features (e.g., safety, traffic volumes, traffic speeds, presence of bicycle lanes, whether the terrain is flat or hilly). In another study, sustainability indicators were assessed where travelled kilometres by bicycle vs. other modes was one key indicator (Buzási and Csete, 2015).

The soft, individual solutions (e.g., personalised route plans) not necessarily increase volume of cycling. Spotswood et al. (2015) analysed cycling as a social practice. They tried to understand the low increasing intensity of cycling in England and the behaviour change towards cycling. They found that there are a lot of stereotypes about everyday bikers ('brave' and 'committed') which determine the general picture about cycling. Respondents deemed that cycling is 'cool' but unattainable because of unsafe roads, storage and lack of showering opportunities. In another study, it has also been found that commuters more likely cycle to work if they have access to indoor bicycle parking facilities (Heinen et al., 2013). During preference specification, the knowledge of the available choices are required. The preferences can be expressed gradually, which is an efficient way to rank a set of choices (Papadakos and Tzitzikas, 2016). Aldred and Jungnickel (2014) discussed differences between locations with established cycling cultures and those with newer cycling cultures. They suggested that substantial infrastructural improvements could and should be made in every place; however, increase of volume of cycling requires different campaigns (e.g., creating arts-led and community projects to leverage social benefits of cycling). 
The description of a route planner application for bikers is mostly technical in the literature. Deeper correspondences, logical connections, personalisation settings are usually not discussed. Su et al. (2010) presented a general description about a route planner application for bikers operated in Vancouver, Canada. They introduced the following personal setting options: distance, elevation gain, safe route features and air pollution. Hochmair and $\mathrm{Fu}$ (2013) presented the general conditions and architecture of their application. This description can be used as a base to develop similar application. Kramers (2014) analysed existing journey planner applications and determined the functions and the requirements towards next generation multimodal traveller information systems. In case of cycling, it has been found that information about bike sharing (available bikes, booking and payment), location of services and navigation are highly required.

\section{Model of biker information management}

Bikers' information management specialities (vs. other transportation modes) are:

- Most of the information is collected before biking.

- Information content of other road users' behaviour has great importance during biking.

- Quick reactions for traffic situations are required.

- Less time and/or less (and also incomplete) information is available for decision making during biking (as bikers are not able to use devices); therefore, decisions are, in the worst case, random made and based on assumptions.

- Movements and behaviour of bikers (body language [e.g., turning sign with hand], metacommunication, mimicry) generate more information than other travellers' movements/behaviour.

During cycling bikers are more reliant on upon their cognitive capabilities due to restricted information availability and short reaction times. Human cognitive capability is defined as follows: recognition and persistent learning capability; being able to create new, reliable, value-added information from ambiguous, incomplete and secondary (tertiary, ...) information sources (particular signs, movement patterns, etc.) using previous experience and existing knowledge. Biker's information management processes have been modelled on Figure 1, where the cognitive methods are highlighted. The information management is discussed on the one hand in general and on the other hand through two typical examples (Table 2):

- Example 1: information service used before riding.

- Example 2: information acquired from other travellers during riding.

In the first example, the biker uses a secondary information because the primary information about the route closure is collected by the background system components and it is then transmitted to the user. In the second example, the biker collects primary information about the behaviour of other traveller directly and so the entire process consists of one less steps. 
Figure 1 Model of biker's information management (see online version for colours)

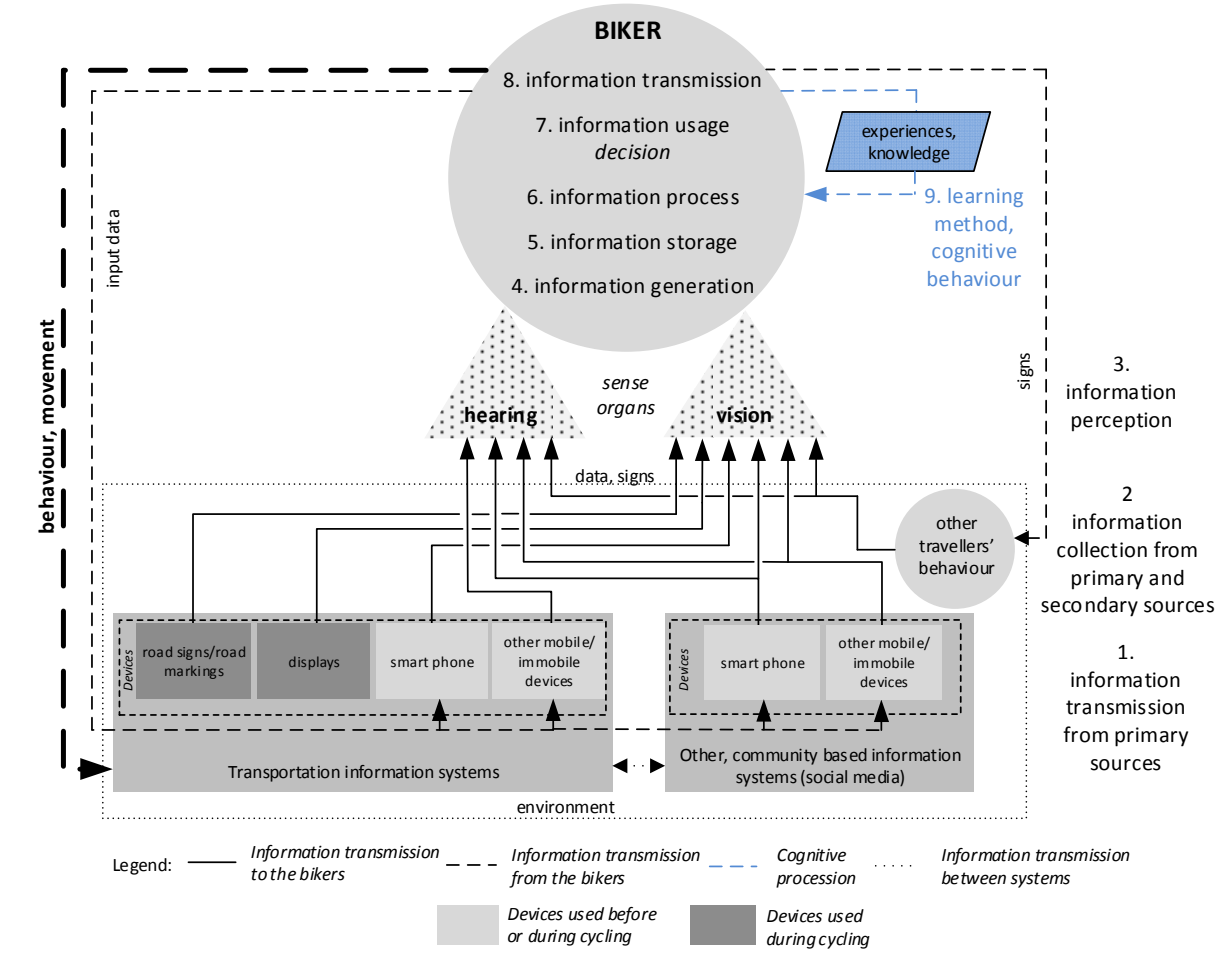

Table 2 Information management - examples

\begin{tabular}{|c|c|c|}
\hline Parts & $\begin{array}{l}\text { Information service } \\
\text { used before riding }\end{array}$ & $\begin{array}{l}\text { Information acquired from } \\
\text { other travellers during riding }\end{array}$ \\
\hline 9 & Avoids the closed route next time & $\begin{array}{l}\text { Expects pedestrian in this point in the } \\
\text { future }\end{array}$ \\
\hline 8 & $\begin{array}{l}\text { Shares the location of the closed road } \\
\text { using social media }\end{array}$ & $\begin{array}{l}\text { The slowed down process is transferred to } \\
\text { other drivers/bikers }\end{array}$ \\
\hline 7 & Selects the most appropriate detour & $\begin{array}{l}\text { Chooses a scenario - slow down, passes } \\
\text { the pedestrian }\end{array}$ \\
\hline 6 & $\begin{array}{l}\text { Considers the closed route during route } \\
\text { planning process (searching for a detour) }\end{array}$ & Considers the situation - scenarios \\
\hline 5 & Stores the information until riding & Processes the information immediately \\
\hline 4 & $\begin{array}{l}\text { Reads the transportation news, perceives } \\
\text { the information }\end{array}$ & Recognises the pedestrian intention \\
\hline 3 & Sees the characters & $\begin{array}{l}\text { Sees the pedestrian's movement } \\
\text { characteristic }\end{array}$ \\
\hline 2 & $\begin{array}{l}\text { Displayed characters on individual } \\
\text { devices }\end{array}$ & $\begin{array}{l}\text { Observes other participants' movements, } \\
\text { behaviour during riding (e.g., pedestrian's } \\
\text { intention to cross the road) }\end{array}$ \\
\hline 1 & $\begin{array}{l}\text { Collection of information about } \\
\text { infrastructure (e.g., closed route) - by } \\
\text { background system components }\end{array}$ & - \\
\hline
\end{tabular}


Phases of the information management:

1 Information collection: bikers use either electronic or conventional mobile/immobile devices before, during or after cycling. The information is either individual (e.g., personal route planning) or collective (e.g., traffic reports). The information sources of electronic devices are either transportation information systems or community-based information systems (social media), where information, experiences (e.g., blog post, Facebook post, tweets) are shared. These infocommunication devices may also be available during cycling (e.g., smart phone attached to the handlebar, smart watch, smart glasses) but only with limited functions. However, in travel phase the installed information devices (e.g., road signs, road markings, displays) have still significant role.

2 Information transmission to bikers: signs, data and voice-based information are received from information devices and other travellers whose movement and behaviour imply/transmit information.

3 Information perception: the received data and signs are perceived by sense organs.

4 Information generation: the information is generated from useful input data and signs.

5 Information storage: the information is stored until utilisation. Duration depends on the decision situation.

6 Information processing: the input information is assessed, classified, selected, etc. Decision alternatives and action plans are created as well as effects of the plans are also considered. Beside data and signs from the environment, the biker's own experience and knowledge are also used.

7 Information usage, decision: the most appropriate alternative or action plan depending on the current situation is chosen. Information management and movements are influenced by this choice. Types of decisions regarding cycling are: decisions with momentary impacts (e.g., reaction for the current traffic situation), decisions with short-term impacts (e.g., route choice), decisions with medium term impacts (e.g., membership of a bike-sharing system) and decisions with long-term impacts (e.g., buy a bicycle).

8 Information transmission from bikers: the result of the decision is an action (movement, behaviour) which may be information source in itself for other participants or for himself/herself (as experience). The action can also be a data input to an infocommunication device either in active way (e.g., sharing information in a community-based information system) or in passive way (e.g., using tracking data).

9 Learning method, cognitive behaviour: the information content of an act is stored as experience or knowledge and may be used for the next processing. 


\section{Situation analysis}

Information services may significantly reduce the negative properties of cycling (e.g., by route offer with few conflict points). We have carried out a situation analysis in order to reveal the positive and negative features of the applications. Special attention has been paid to route planner applications.

Figure 2 Classification of information services for bikers

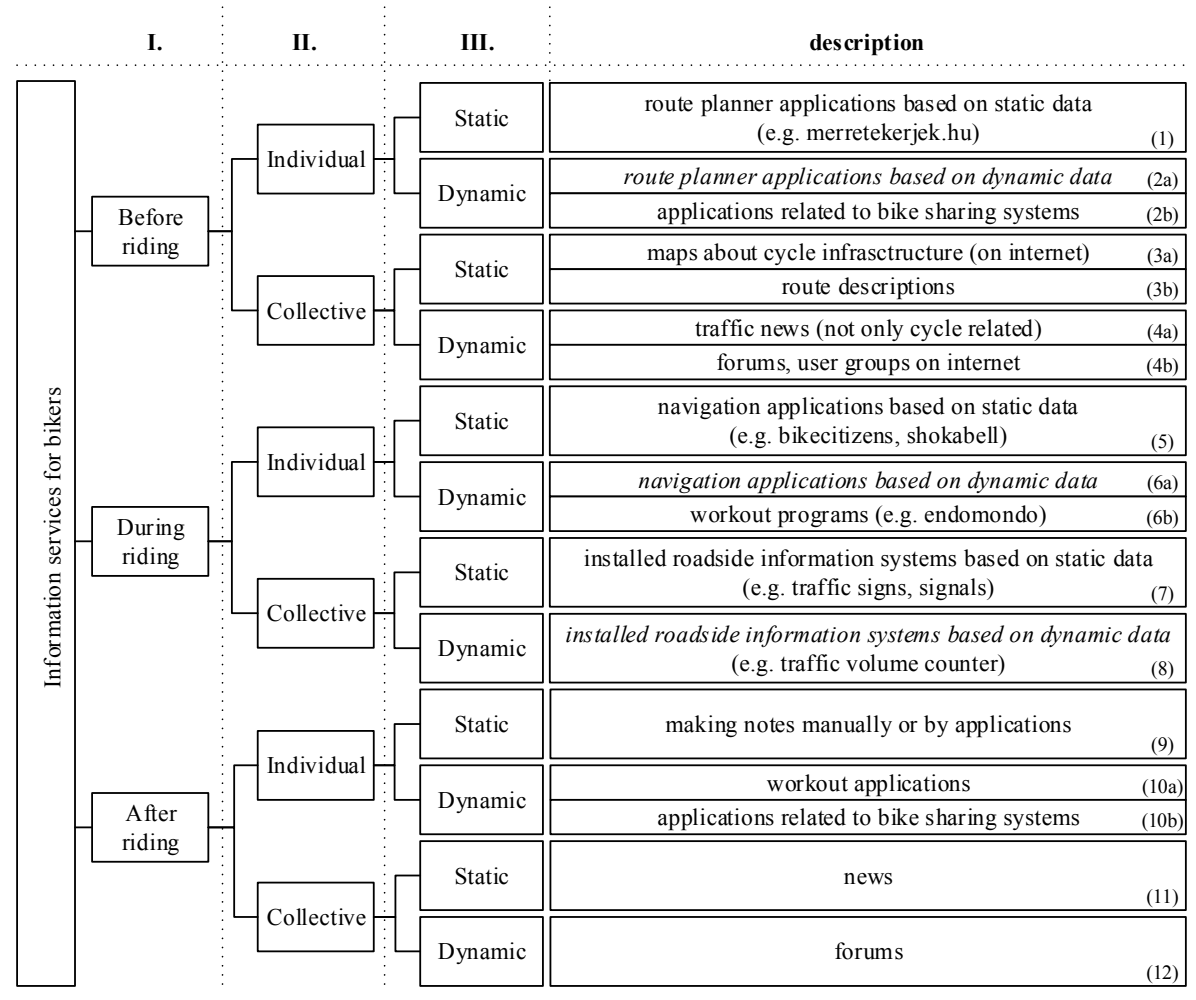

Note: Barely introduced services

\subsection{Information services for bikers}

We have classified the services according to:

1 phase of use (before, during or after riding)

2 scope of users (individual, collective)

3 used data (static, dynamic). 
The services are operated on either mobile or immobile devices that can be the following according to scope of users:

- Individual: mobile (e.g., smart phone, smart watch, smart glasses)/immobile (e.g., desktop).

- Collective: mobile (e.g., portable VMS - variable message sign)/immobile (e.g., fix VMS, displays).

The classification is summarised in Figure 2. Italic letters indicate barely introduced services. Route planner applications have been analysed in details.

Before riding route planner applications based on static data can be used (1). The route planner applications based on dynamic data (e.g., weather data, traffic volume) for bikers (2a) are not widespread currently. Applications related to bike sharing systems (2b) are mostly operated with dynamic data (e.g., number of free bicycles, free docking stations). The advanced applications provide also value-added information (e.g., Bycyclen application in Copenhagen provides route planning/navigation and information about transfer opportunities). Static web or paper-based maps (3a) and route descriptions (3b) provide collective information. Dynamic, collective information sources include traffic related news (4a) and internet forums (4b).

During riding the location-based navigation services can provide information. However, currently only a few navigation services based on mainly static data are available for bikers (5). There is huge development potential in the navigation services based on dynamic data (6a). Work out applications (6b) provide dynamic services as they track the users' position. However, these applications are used mostly for evaluating the workout. Collective information is presented on installed roadside tables and signs (7a) and via VMS devices (7b). VMS is not a widespread information source in cycle traffic yet. Cycle traffic counter devices are widely implemented on roads; however, these devices provide information not primarily for the bikers.

After riding the biker can make notes about riding (9) or can evaluate it using workout application (10a). After usage of bike sharing system, its application can inform the user about the details of the hiring (e.g., covered distance, price) (10b). Bikers may collect information from transport related news (11) or forums where bikers' experiences are compiled and shared (12).

\subsection{Route planner applications}

Bikers, similarly as other travellers, also use route planner applications for preparing riding. The applications can be also used during riding to re-plan the trip, but they do not provide navigation services, as they do not track the user.

We have systematically determined the most relevant points of view of analysis. The description of operational characteristics of the applications (e.g., development specifications) was not available for us. 
Table 3 Analysis of route planner applications for bikers (results based on data of 2016 summer)

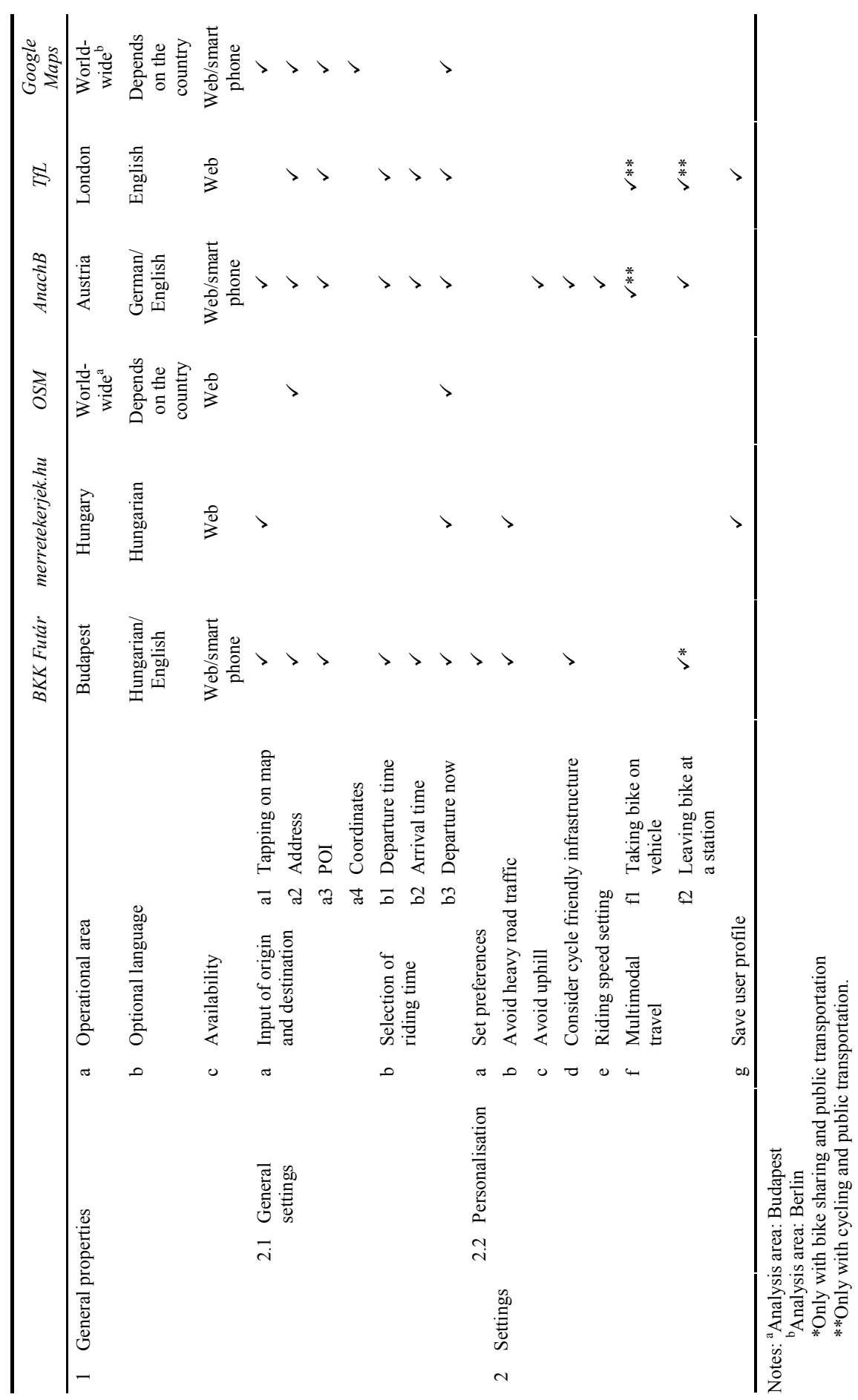


Personalised information services for bikers

Table 3 Analysis of route planner applications for bikers (results based on data of 2016 summer) (continued)

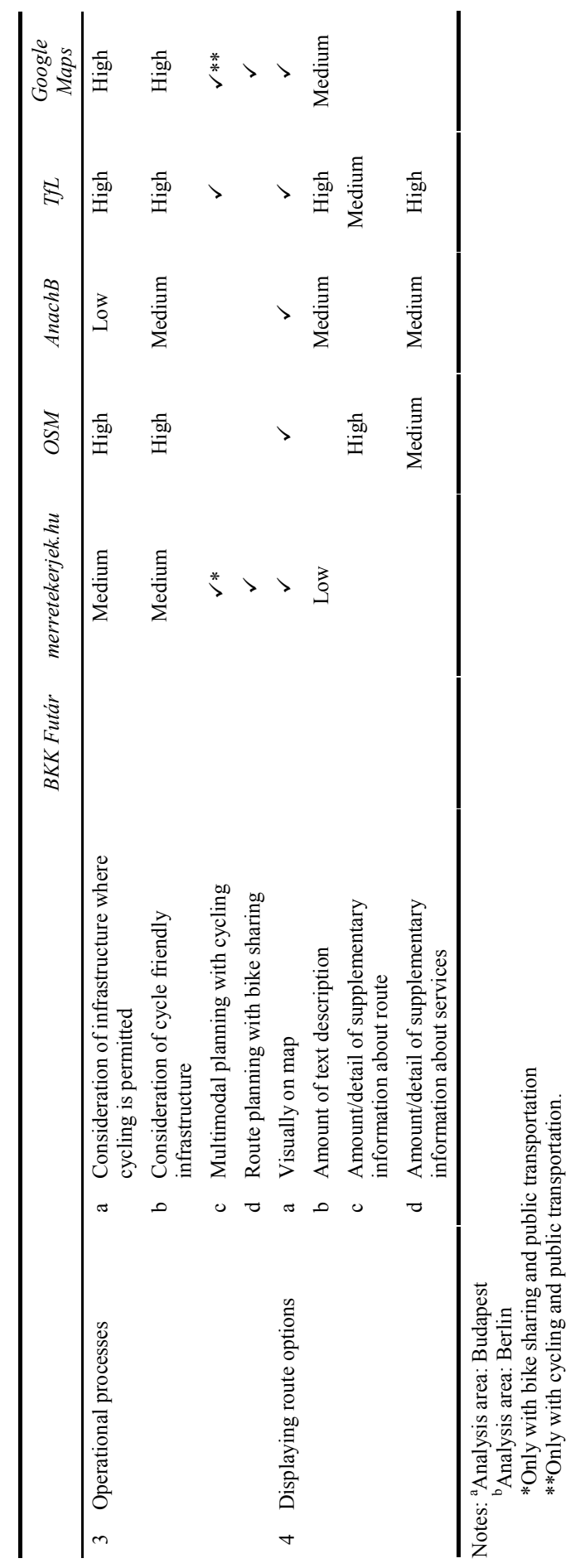


Two popular Hungarian and four well-known and widely used foreign route planner applications have been chosen to analyse the current situation. Operational area of the foreign applications is very similar to Budapest (Hungary) concerning the cycling conditions (metropolis, increasing rate of cycling in modal share, etc.). The selected applications:

- BKK Futár [futar.bkk.hu]: operated by the Centre for Budapest Transport. It provides route planning for public transport users and bikers. The service is based on real-time data regarding both public transportation and Budapest bike-sharing system (BuBi) (e.g., temporary closed stations). The multimodality is realised partially as combination of bike sharing and public transportation.

- merretekerjek.hu: pioneer Hungarian cycling route planner application developed by private community.

- Open street map (OSM) [openstreetmap.org]: freely editable map with cycling submodule. It is not able to plan multimodal routes.

- AnachB [anachb.at]: pioneer Austrian multimodal route planner application, which covers the whole country. It is operated by a transport association.

- TfL [tfl.gov.uk]: operated by Transport for London. It provides route planning for public transport users. The service based on real-time data. The multimodality is realised partially as combination of cycling and public transportation.

- Google Maps [googlemaps.com]: well-known and world-wide used route planner application which has cycling route planner submodule covering several countries.

Several origin-destination queries have been analysed in order to draw conclusions about the operation (e.g., considering cycle friendly infrastructure). The results of the analysis are summarised in Table 3.

Several statistics about users, usage of applications and ratings are available as well as user can give feedbacks too. In case of BKK Futár the available statistics are: number of downloads (over 150 thousand), number of usage (over 1 million) and number of route plans (over 300 thousand). Similar statistics are also available about other applications; however, these data are mostly too general, not specified for cycling. We have reviewed these statistics in order to substantiate the statements. The smartphone applications are mostly rated with high scores, which reflects that users are usually rather satisfied with them.

We have identified the most pioneer and exemplary properties that should be realised by the 'ideal' application.

1 General settings:

$\checkmark \quad$ smart phone application covers the same functions as the web-based application (BKK Futár, AnachB).

\section{Personalisation:}

$\checkmark$ planning only with cycle friendly roads or fastest roads (where not only the cycle friendly roads are considered) (BKK Futár)

$\checkmark$ excluding dirt roads, avoiding heavy road traffic (merretekerjek.hu) 
$\checkmark \quad$ avoiding uphill, preferring cycle roads, setting riding speed (AnachB)

$\checkmark$ multimodal travel with taking bike on vehicle or leaving bike at a station (AnachB, TfL)

$\checkmark \quad$ using bike sharing systems (BKK Futár, TfL).

3 Operational processes:

$\checkmark$ detailed consideration of cycle infrastructure (merretkerjek.hu, AnachB)

$\checkmark$ consideration of zebra crossings (with taking the bike) (BKK Futár)

$\checkmark$ multimodal planning combining public transportation and cycling (TfL)

$\checkmark \quad$ multimodal planning combining public transportation and bike sharing (BKK Futár)

$\checkmark \quad$ complete multimodal planning (combining cycling, bike sharing, public transportation, car usage, car sharing) (AnachB).

4 Displaying route options:

$\checkmark$ road type (bike lane, bike road, etc.), elevation profile (merretekerjek.hu, AnachB)

$\checkmark$ detailed text description (AnachB)

$\checkmark$ denoting surrounding cycling infrastructure (merretekerjek.hu, OSM)

$\checkmark$ supplementary information about services (AnachB - service points, bike stands).

We have also ascertained the weaknesses of the existing applications:

$\times$ especial road planning for bikers (completely mapping cycle infrastructure and considering cycle friendly infrastructure)

$\times$ only few personalisation settings

$\times$ neglecting static (e.g., potholes) and dynamic (e.g., icy road) properties of the roads.

\section{Data collection}

Data have been collected about bikers' habits and expectations towards information services by questionnaire survey. One question regards to one aspect of the analysis. The considered aspects belong to personal attributes or basic process or information management. Structure of the collected data is presented in Figure 3. The aspects (indicated by 'boxes') are described by list of options. Aspects are denoted with two digits; options are denoted with three digits where the first two digits refer to the number of the aspect (interpretation of linking lines can be found at the end of Section 7).

Two types of aspects have been identified. Accordingly, they have been assessed in two different ways:

- Either option is selected from the list - indicated by blue colour. Respondents chose one option (or in case of information sources /15/perhaps more), which represents his/her attribute or preference. 
- Every option from the list is assessed by an importance factor (IF) - indicated by red colour.

Respondents chose an IF which reflects their habit/preference, namely, how the option is important for them (so-called Likert-scale). The IF is placed either on 1-3 (1: not important, 2: important, 3: highly important) or on 1-5 scale (1: not important, 2: less important, 3: indifferent, 4: important, 5: highly important).

The questionnaire was developed by system and process oriented approach taking into consideration structure and content of other surveys. Each respondent grasps the content of questions in different manner (Hosseininasab, 2015), therefore clear formulation of questions is important to reduce the distance of the provided answers. The goal is to minimise the number of biased answer which may indirectly influence the outcome of the survey (Roy et al., 2016).

Figure 3 Structure of the collected data - strength of correspondences between aspects pertaining to 'urban biker' type (see online version for colours)

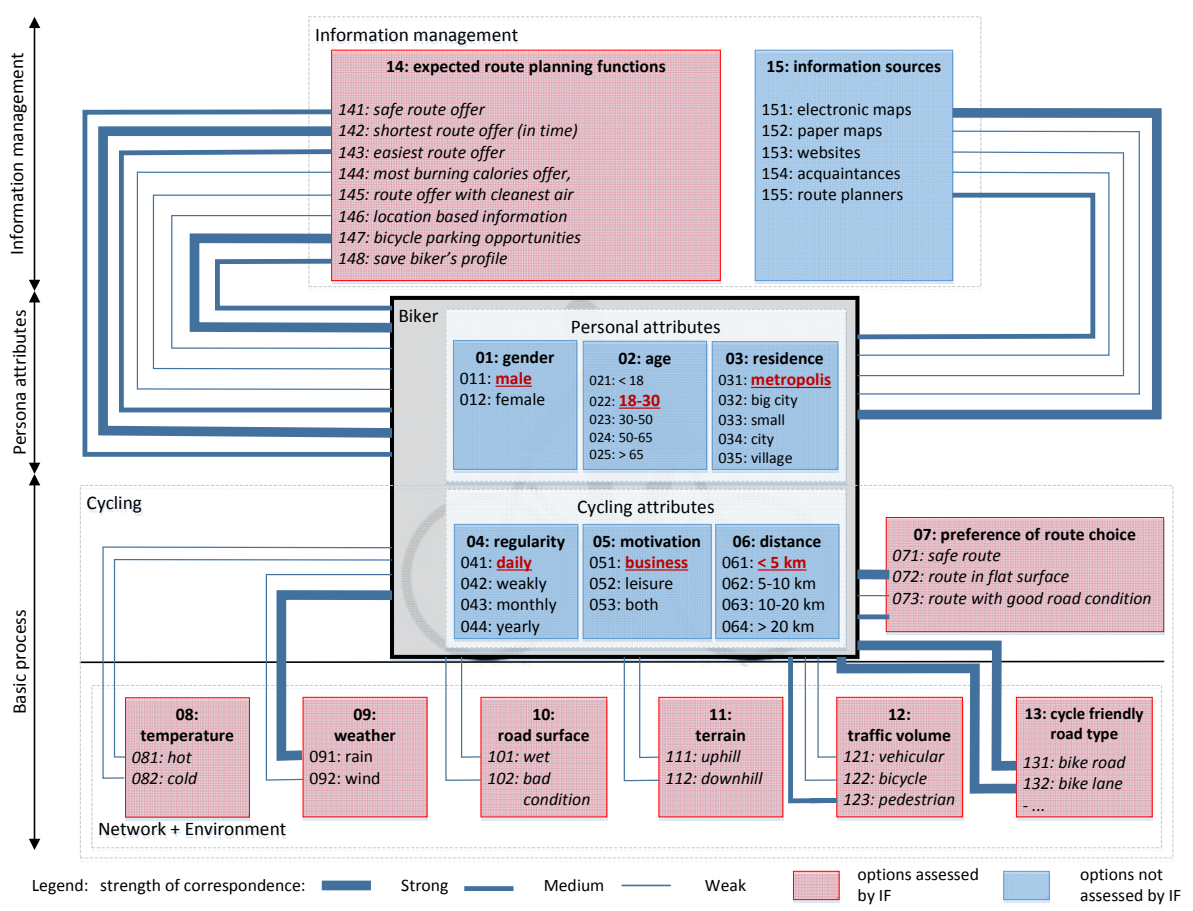

After compilation of the questionnaire, it was tested by focus group to check the appropriateness of the questions. The members were selected from our university students and experienced colleagues. Considering the comments (e.g., not preferring scale questions, preferring multiple choices and picture illustrated questions) the questionnaire was modified.

The comprehensive survey was performed via internet in one week in October 2015. 770 responses have been received. The respondents were selected by snowball method 
when members of a certain group with similar attributes answer. The questionnaire was promoted on websites and social pages of the most relevant Hungarian cycling communities as well as among university students. University students are a target group of cycling as most of them are environmental conscious, are in good physical condition and cannot afford other individual travel modes. Accordingly, majority of respondents were members of such a cycling or university community/group. However, in order to confirm the results on a more representative, larger population covering more potential bikers we are going to perform a comprehensive survey based on these experiences. In this way respondents' bias can be omitted.

\section{Analysis method}

The correspondences have been revealed either between options or between options and aspect or between aspects according to aggregation levels. Our elaborated analysis method has been presented on 5th IEEE International Conference on Advanced Logistics and Transport (IEEE ICALT'2016) (Földes and Csiszár, 2016). As results, two-dimensional matrices are produced. Content of the matrices depends on the aggregation level and types of paired aspects/options. At first, in the 'lower level approach', the options (I.) then, in the 'higher level approach', the aspects were investigated (II.) pairwise.

\subsection{Correspondences between options}

Options of two different aspects (in general aspect 1 and aspect 2) have been investigated in pairs in order to reveal the correspondence between them. The result is the strengthness index (SI). Three types of correspondences have been identified considering whether the option is assessed by IF or not:

a options are not assessed by IF

b only one option is assessed by IF

c both options are assessed by IF.

In case of type A all options of the two aspects are considered at the same time. Steps:

1 Create a matrix where the column and row headers are the options.

2 Count the number of responses in each cell: $x_{i j}$ (where $i$ options of aspect $1, j$ options of aspect 2). $x_{i j}$ represents number of responses where both options are chosen.

3 Sum the number of responses in each column: $X_{j} . X_{j}$ represents total number of responses belonging to $j$ option. (Similarly, $X_{i}$ can be also calculated.)

4 Calculate the $S I_{i j}$ :

$$
S I_{i j}=\frac{x_{i j}}{X_{j}} \cdot 100[\%]
$$


The more respondents select both options, the strengthness index is higher. $S I_{i j}$ is described by categories: strong $S I_{i j}>75 \%$, medium $75 \%>S I_{i j}>50 \%$ and weak $S I_{i j}<50 \%$.

In case of type B, all options of aspect 1 and only one option of aspect 2 (assessed by IF) are considered at the same time. Steps of calculation:

1 Create a matrix where column headers are options of aspect 1 and row headers are IFs of only one selected option of aspect 2.

2 Create cross-table queries, which count the number of responses in each cell $\left(x_{i j}\right)$ (where $i$ options of aspect $1, j$ IFs of option of aspect 2).

3 Assign weight number for each cell $\left(w_{i j}\right)$ according to scale type. The appropriate

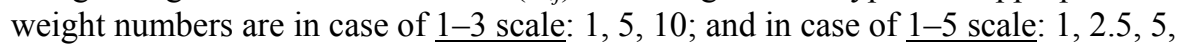
$7.5,10$.

4 Calculate weighted number of responses $\left(z_{i j}\right)$ :

$$
z_{i j}=x_{i j} \cdot w_{i j}
$$

5 Calculate $S I_{i j}$ : sum of weighted number of responses in row divided by sum of responses in row:

$$
S I_{i j}=\frac{\sum_{j} z_{i j}}{\sum_{j} x_{i j}}
$$

In case of type $\mathrm{C}$, only one option of aspect 1 and only one option of aspect 2 are considered at the same time. Steps of calculation:

1 Create a matrix, where the column and row headers are IFs of only the selected options of aspect 1 and aspect 2 .

2 Create cross-table queries, which count the number of responses in each cell $\left(x_{i j}\right)$ ( $i$ IFs of the selected option of aspect $1, j$ IFs of the selected option of aspect 2 ).

3 Assign weight number for each cell $\left(w_{i j}\right)$. It was necessary to determine weight numbers in every possible combination according to the scales of IF $(3-3,3-5,5-5)$. We have found a scale with 10 elements to be the most appropriate because in this way, the comparison of different combinations is more remarkable (Tables 4-6).

4 Determine $z_{i j}$ - similar than in case of type B.

5 Calculate $S I_{i j}$ : sum of weighted number of responses divided by sum of responses:

$$
S I_{i j}=\frac{\sum_{i j} z_{i j}}{\sum_{i j} x_{i j}}
$$

In case of type B and C, $S I_{i j}$ is also expressed by categories: strong $S I_{i j}>7.5$, medium $7.5>S I_{i j}>5$ and weak $S I_{i j}<5$. In order to produce comparable results, the weight numbers $\left(w_{i j}\right)$ were chosen between 1 and 10 in cases B and C. 
Table 4 Weight numbers in correspondence type C (3-3)

\begin{tabular}{lllcc}
\hline \multirow{2}{*}{$w_{i j}$} & \multicolumn{3}{c}{ Option 2} \\
\cline { 3 - 5 } & & 1 & 2 & 3 \\
\hline \multirow{2}{*}{ Option 1} & 1 & 1 & 3 & 6 \\
& 2 & 3 & 5 & 8 \\
& 3 & 6 & 8 & 10 \\
\hline
\end{tabular}

Table 5 Weight numbers in correspondence type C (3-5)

\begin{tabular}{lllllll}
\hline \multirow{2}{*}{$w_{i j}$} & \multicolumn{5}{c}{ Option 2} \\
\cline { 2 - 7 } & 1 & 2 & 3 & 4 & 5 \\
\hline \multirow{2}{*}{ Option 1} & 1 & 1 & 2 & 3 & 4 & 5 \\
& 2 & 2 & 4 & 5 & 6 & 7 \\
& 3 & 3 & 5 & 7 & 9 & 10 \\
\hline
\end{tabular}

Table 6 Weight numbers in correspondence type C (5-5)

\begin{tabular}{lllllll}
\hline \multirow{2}{*}{$w_{i j}$} & \multicolumn{5}{c}{ Option 2} \\
\cline { 3 - 7 } & 1 & 2 & 3 & 4 & 5 \\
\hline Option 1 & 1 & 1 & 2 & 3 & 4 & 5 \\
& 2 & 2 & 3 & 4 & 5 & 6 \\
& 3 & 3 & 4 & 5 & 6 & 8 \\
& 4 & 4 & 5 & 6 & 7 & 9 \\
& 5 & 5 & 6 & 8 & 9 & 10 \\
\hline
\end{tabular}

\subsection{Correspondences between aspects}

By two-tier aggregation of results regarding options, more synthetic indicators can be prepared.

$1 \quad$ Average of $S I_{i j}$ either by $i$ or $j$. These indices (aggregated SIs by either row or column) refer to strength of correspondence between one aspect and one option of another aspect.

2 Average either row or column aggregated SIs. The totally aggregate index refers to the strength of correspondence between two aspects.

The first level aggregation can be applied in cases of all types; however, the second level aggregation is to be calculated in cases of type B and C. 


\section{Results}

In tables of results, the same colouration has been applied as in Figure 3. Options assessed with IF are red, the remaining ones are blue. Table 7 presents an excerpt of results of correspondence type A. It shows, how options of regularity / $04 /$ and motivation $/ 05 /$ relate to options of information sources $/ 15 /$. For instance: $81.6 \%$ of daily bikers use route planner applications /042-155/. Different colours (strong: dark grey, medium: light grey, weak: white) indicate the strength of the correlation. The aggregated SIs are presented in frame.

As aspect $/ 15 /$ is assigned to a multiple question, the sum of SIs according to column is not $100 \%$. (All the remaining aspects without IFs are assigned to simple question, therefore in those cases the column sum is $100 \%$ ).

Table 8 presents an excerpt of results of correspondence type B and C. For instance:

- SI between option and aspect (first step of aggregation): between avoid rain /091/ and regularity /04/ the aggregated row SI is 7.1 .

- SI between aspects (second step of aggregation): between weather/09/ and regularity $/ 04 /$ the aggregated SI is 6.4.

Based on the combination of the options of biker's direct aspects /aspects 01-06/ several biker types are to be defined. In order to prove the applicability of the analysis method, we have identified some typical biker types and the SIs have been calculated. For illustration purposes the 'urban biker' type (gender: male, age: 18-30, residence: metropolis, regularity: daily, motivation: business, distance: $<5 \mathrm{~km}$ ) has been selected. Strength of correspondences (SIs) in case of 'urban biker' are also presented in Figure 3. The thickness of the lines represents the strength. For instance: 'urban biker' expects shortest route offer /142/ and bicycle parking opportunities /147/ as route planning functions. They try to avoid rainy weather $/ 091 /$ and they like to use bike road $/ 131 /$ and bike lane $/ 132 /$.

Table 7 Results of type A correspondence - excerption

\begin{tabular}{|c|c|c|c|c|c|c|}
\hline \multirow[b]{2}{*}{$S I_{i j}$} & & \multicolumn{5}{|c|}{ 15: information sources } \\
\hline & & $\begin{array}{c}151: \\
\text { maps } \\
\text { (electronic) }\end{array}$ & $\begin{array}{l}\text { 152: } \\
\text { maps } \\
\text { (paper) }\end{array}$ & $\begin{array}{c}\text { 153: } \\
\text { websites }\end{array}$ & $\begin{array}{c}\text { 154: } \\
\text { acquaintances }\end{array}$ & $\begin{array}{c}155: \\
\text { route } \\
\text { planners }\end{array}$ \\
\hline \multirow{5}{*}{$\begin{array}{l}04: \\
\text { regularity }\end{array}$} & 042: daily & 55.8 & 19.8 & 43.0 & 43.0 & 81.6 \\
\hline & 043: weekly & 74.2 & 21.8 & 54.0 & 46.8 & 80.7 \\
\hline & 044: monthly & 78.2 & 17.8 & 54.7 & 47.1 & 80.4 \\
\hline & 045: yearly & 74.4 & 24.6 & 52.8 & 47.8 & 78.0 \\
\hline & Aggregated & 70.7 & 21.0 & 51.1 & 46.2 & 80.2 \\
\hline \multirow{4}{*}{$\begin{array}{l}05: \\
\text { motivation }\end{array}$} & 051: business & 75.3 & 25.3 & 57.7 & 48.9 & 78.4 \\
\hline & 052: leisure & 73.5 & 13.6 & 43.5 & 41.2 & 75.7 \\
\hline & 053: both & 70.1 & 21.7 & 51.2 & 48.3 & 85.1 \\
\hline & Aggregated & 73.0 & 20.2 & 50.8 & 46.1 & 79.7 \\
\hline
\end{tabular}

Notes: Strength of correlation: strong, medium, weak 
Personalised information services for bikers

Table 8 Results of type C correspondence - excerption (see online version for colours)

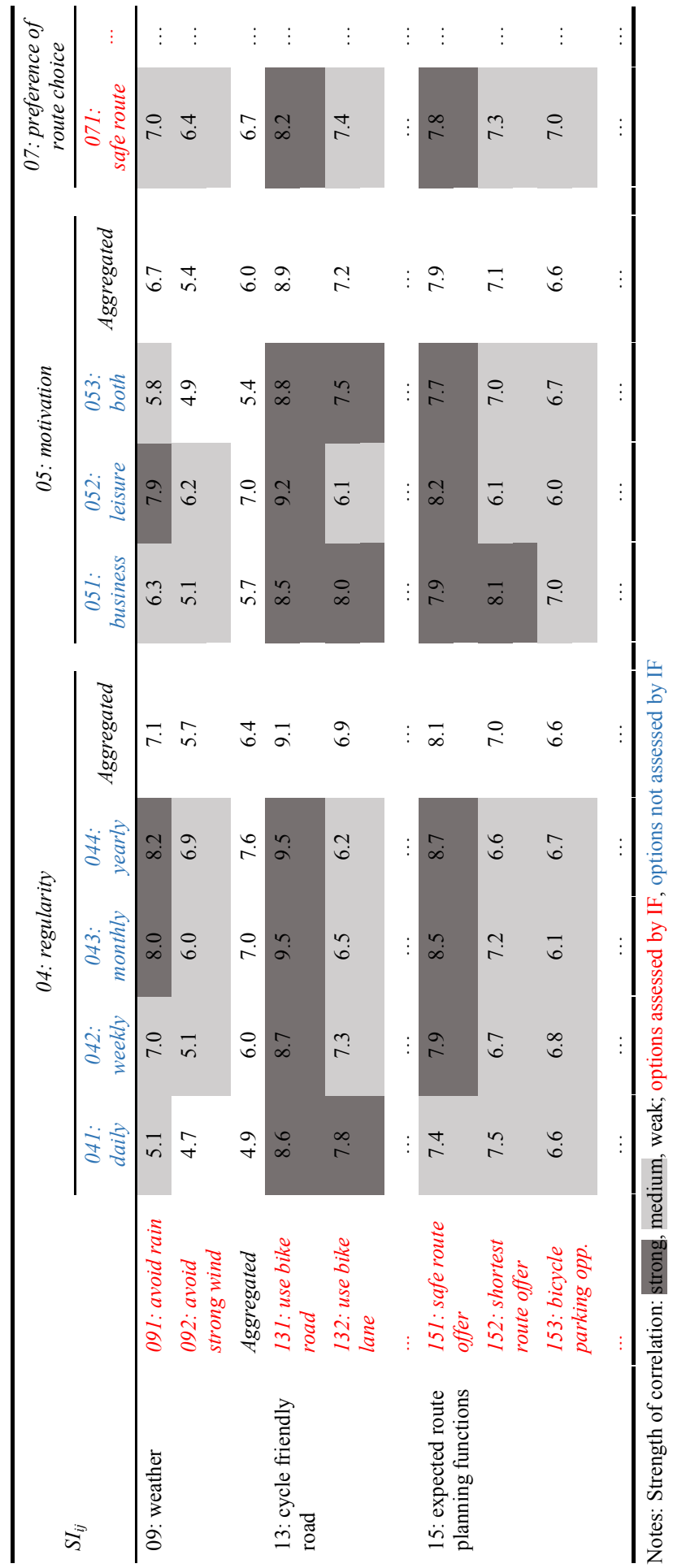




\section{Discussion}

The results proved several preliminary hypotheses about cycling community. The most relevant hypotheses and their confirmation have been summarised. The hypotheses regard to correspondences between option-option (SI) and option-aspect (aggregated SI). Some hypotheses are represented in Tables 7 and 8.

The motivation /05/ significantly influences the expected route planning functions /14/. The correspondence between shortest route offer /142/ and business motivation $/ 051 /$ is extremely strong (8.1), in case of leisure motivation /052/ is less (6.1). The leisure motivated /052/ bikers principally require safe route offer $/ 141 /$ (8.2). The different between the mentioned motivations in case of information about bicycle parking opportunities /143/ is also obvious (business motivation: 7.0, leisure motivation: 6.0). Explanation: The business-motivated bikers especially prefer time savings. The correspondences between type of residence $103 /$ and expected route planning functions 14/ are also significant. Bikers in small cities $(<100.000$ habitants) /033/ require safe route offer $/ 141 /(8.3)$ and route offer with cleanest air $/ 145 /(6.5)$. The air quality is not so important (5.5) for bikers in metropolises $/ 031 /$, despite the fact that the air is more polluted in big cities.

The initial hypothesis has been also confirmed by results. Only one fourth of the respondents do not use any route planner application before riding. However, if route planner application using real-time data existed, $25.7 \%$ of the respondents would use it every time before riding; $42.9 \%$ would use it only in case of unfamiliar route/destination and only $9 \%$ would never use it. Half of the respondents consider as most significant disadvantage of the existing route planner applications that they cannot plan routes especially for bikers. Using route planner application /155/ depends on the cycling distance /06/. The longer the distance, the more bikers use application (85\% of bikers use an application /155/ if the distance is more than $20 \mathrm{~km} / 064 /$ ). Explanation: for longer distances biker's experience, knowledge is less typical. The motivation /04/ and regularity $/ 05 /$ do not influence usage of route planner applications /155/ - proportion is rather high. Explanation: the route planning is important for all bikers.

Preference of route choice /07/ depends on the attitudes towards network and environment /08-12/. There are strong correlations between safe route choice /071/ preference and avoiding bad weather conditions (rainy /091/7.1, windy /092/ 6.4) or bad road conditions $/ 102 /(6.2)$. There is also strong correlation between preference of bike $\mathrm{road} / 131 /$ and bike lane $/ 132 /$ and preference of safe route choice /071/ (8.2, 7.4). Explanation: bikers feel the rainy and windy weather as unsafe condition. However, between safe route offer $/ 071 /$ and wet road surface /101/ there is not strong correlation (5.1). There are strong correlations between certain biker attributes and preferred network/environment conditions. Women /012/ are more sensitive to bad weather conditions $/ 09 /$, but less sensitive to road conditions $/ 10 /$. The bikers cycling rarely (yearly) /044/ try to avoid uphill /111/ (5.0), cold /082/ (8.1) and rainy /091/ (6.3) weather. The daily bikers $/ 041 /$ are not bothered from uphill $/ 111 /$ (3.2) or from cold weather /082/ (3.1).

The survey contained another question regarding the weaknesses of current route planner applications. Without further detailed analysis and discussion, we present some relevant facts that are rather coincident with the results of our situation analysis: 
- the most disturbing property is the lack of especial route planning for bikers (by $50 \%$ of respondents)

- the second one is that applications neglect static and dynamic properties of the roads (by $23 \%$ of respondents).

\section{Conclusions}

The main contributions of the research are:

- model of biker's information management

- classification of biker information services

- determination of the most relevant points of view of analysis for route planner applications

- analysis method to determine the strength of correspondences between bikers' mobility and information management attributes.

Existing information services have been analysed in order to determine exemplary functions and properties. It has been found that current route planner applications have only a few personalisation settings, neglect properties of road and lack especial route planning for bikers. We have applied the elaborated method using data of our questionnaire survey. Some calculation results regarding a selected urban biker type have been presented for illustration purposes. The research results can be used during development of an adaptive, personalised, decision supporting information application where only some data input from the biker (to recognise the type of biker) is enough to provide adequate personalised information. In this way, the long (re)setting processes can be significantly shortened.

We faced, as lessons learnt:

- Information services and applications are ever altering, therefore neither the evaluation aspects nor the results of the evaluation are permanent.

- Evaluation of applications by 'trials', without knowledge about their background operational properties is rather challenging, but the results are reliable.

- Adequate structure and content of the questionnaire could be deducted from the required outputs, in order to be able to draw relevant conclusions.

- Identifying relevant correspondences between aspects and options required system and process oriented approach, experience in analysis of other transportation modes and last, but not least everyday practice of cycling.

Our future research focuses on similar researches of other passenger transportation modes. We aimed at both examination of the available information services and revealing the correspondences between mobility and information management attributes in case of all modes. Our aim is to facilitate the creation of multimodal traveller assistant (guiding) services. 


\section{References}

Aldred, R. and Jungnickel, K. (2014) 'Why culture matters for transport policy: the case of cycling in the UK', Journal of Transport Geography, Vol. 34, pp.78-87, DOI: 10.1016/j.jtrangeo.2013.11.004.

Ana, S., Pinto, I., Ribeiro, D. and Delgado, J. (2014) 'Multicriteria analysis for evaluation of bike lane routes integrated to public transportation', Procedia - Social and Behavioral Sciences, Vol. 162, pp.388-397, DOI: 10.1016/j.sbspro.2014.12.220.

Bhat, C.R., Dubey, S.K. and Nagel, K. (2015) 'Introducing non-normality of latent psychological constructs in choice modeling with an application to bicyclist route choice', Transportation Research Part B: Methodological, Vol. 78, pp.341-363, DOI: 10.1016/j.trb.2015.04.005.

Braun, L.M., Rodriguez, D.A., Cole-Hunter, T., Abros, A., Donaire-Gonzalez, D., Jerett, M., Mendez, M.A., Nierwenhuijsen, M.J. and de Nazelle, A. (2016) 'Short-term planning and policy interventions to promote cycling in urban centers: findings from a commute mode choice analysis in Barcelona, Spain', Transportation Research Part A: Policy and Practice, Vol. 89, pp.164-183, DOI: 10.1016/j.tra.2016.05.007.

Buzási, A. and Csete, M. (2015) 'Sustainability indicators in Afmultimssessing urban transport systems', Periodica Polytechnica Transportation Engineering, Vol. 43, No. 1, pp.138-145, DOI: 10.3311/PPtr.7825.

Cserháti, B. and Csiszár, Cs. (2016) 'Conception of personalized parking assistant application', Periodica Polytechnica, Civil Engineering, Vol. 60, No. 2, pp.181-188, DOI: 10.3311/PPci.7679.

Ehrgott, M. Wang, J.Y.T., Raith, A. and Houtte, C. (2012) 'A bi-objective cyclist route choice model', Transportation Research Part A: Policy and Practice, Vol. 46, No. 4, pp.652-663, DOI: $10.1016 / \mathrm{j}$.tra.2011.11.015.

Földes, D. and Csiszár, Cs. (2015) 'Route plan evaluation method for personalized passenger information service', Transport, Vol. 30, No. 3, pp.273-285 (Special Issue on Smart and Sustainable Transport), DOI: 10.3846/16484142.2015.1086889.

Földes, D. and Csiszár, Cs. (2016) 'Revealing correspondences between bikers' mobility and information management attributes', 5th IEEE International Conference on Advanced Logistics and Transport (ICALT2016).

Habib, K.N., Mann, J., Mahmoud, M. and Weiss, A. (2014) 'Synopsis of bicycle demand in the City of Toronto: investigating the effects of perception, consciousness and comfortability on the purpose of biking and bike ownership', Transportation Research Part A: Policy and Practice, Vol. 70, pp.67-80, DOI: 10.1016/j.tra.2014.09.012.

Heinen, E., Maat, K. and van Wee, B. (2013) 'The effect of work-related factors on the bicycle commute mode choice in the Netherlands', Transportation, Vol. 40, No. 1, pp.23-43, DOI: $10.1007 / \mathrm{s} 11116-012-9399-4$.

Hochmair, H. and Fu, J. (2013) Web Based Bicycle Trip Planning for Broward Country, Florida Report, GIS Center, Forida International University, Florida [online] $\mathrm{http}: / /$ digitalcommons.fiu.edu/gis/2/ (accessed 18 August 2016).

Hosseininasab, A. (2015) 'Regularisation and reliability assessment of data in survey analysis', International Journal of Data Analysis Techniques and Strategies, Vol. 7, No. 3, pp.284-300, DOI: 10.1504/IJDATS.2015.071368.

Karádi, D., Nagy, E. and Csiszár, Cs. (2015) 'Integrated information application on mobile devices for air passengers', 4th International Conference on Models and Technologies for Intelligent Transportation Systems (MT-ITS 2015), Budapest, Hungary, pp.304-311, DOI:10.1109/MTITS.2015.7223272.

Kramers, A. (2014) 'Designing next generation multimodal traveler information systems to support sustainability oriented decisions', Environmental Modelling \& Software, Vol. 56, pp. 83-93, DOI: 10.1016/j.envsoft.2014.01.017. 
Papadakos, P. and Tzitzikas, Y. (2016) 'Comparing the effectiveness of intentional preferences versus preferences over specific choices: a user study', International Journal of Information and Decision Sciences (IJIDS), Vol. 8, No. 4, pp.378-403, DOI: 10.1504/IJIDS.2016.10001391.

Passafaro, P., Rimano, A., Piccini, M.P., Metastasio, R., Gambardella, V., Gullace, G. and Lettieri, C. (2014) 'The bicycle and the city: desires and emotions versus attitudes, habits and norms', Journal of Environmental Psychology, Vol. 38, pp.76-83, DOI: 10.1016/j.jenvp.2013.12.011.

Roy, A., Tavana, M., Banerjee, S. and Di Caprio, D. (2016) 'A secured context-aware tourism recommender system using artificial bee colony and simulated annealing', International Journal of Applied Management Science (IJAMS), Vol. 8, No. 2, pp.93-113, DOI: 10.1504/IJAMS.2016.077014

Santos, G., Maoh, H., Potoglou, D. and von Brunn, T. (2013) 'Factors influencing modal split of commuting journeys in medium-size European cities', Journal of Transport Geography, Vol. 30, pp.127-137, DOI: 10.1016/j.jtrangeo.2013.04.005.

Spotswood, F., Chatterton, T., Tapp, A. and Williams, D. (2015) 'Analysing cycling as a social practice: an empirical grounding for behaviour change', Transport Research Part F: Traffic Psychology and Behaviour, Vol. 29, pp.22-33, DOI: 10.1016/j.trf.2014.12.001.

Stinson, M.A. and Bhat, C.R. (2005) 'A comparison of the route preferences of experienced and inexperienced bicycle commuters', TRB 2005, pp.05-1434.

Su, J.G., Winters, M., Nunes, M. and Brauer, M. (2010) 'Designing a route planner to facilitate and promote cycling in Metro Vancouver, Canada', Transportation Research Part A: Policy and Practice, Vol. 44, No. 7, pp.495-505, DOI: 10.1016/j.tra.2010.03.015.

Tilahun, N.Y., Levinson, D.M. and Krizek, K.J. (2007) 'Trails, lanes, or traffic: valuing bicycle facilities with an adaptive stated preference survey', Transportation Research Part A: Policy and Practice, Vol. 41, pp.287-301, DOI: 10.1016/j.tra.2006.09.007.

Winters, M., Davidson, G., Kao, D. and Teschke, K. (2010) 'Motivators and deterrents of bicycling: comparing influences on decisions to ride', Transportation, Vol. 38, No. 1, pp.153-168, DOI: 10.1007/s11116-010-9284-y. 